\title{
A NOTE ON THE RELATIONSHIP BETWEEN CERTAIN SUBGROUPS OF A FINITE GROUP
}

\author{
W. E. DESKINS
}

A well-known result of G. Frobenius (cf. [2]) states that if $\mathfrak{F C}$ is a normal subgroup of the finite group $\mathcal{G}$, then an irreducible $\mathcal{G}$-module (relative to any base field $\mathfrak{F}$ ) either remains irreducible as an $\mathcal{H}$-module or decomposes into a direct sum of conjugate irreducible $\mathfrak{H}$-modules. Simple examples readily demonstrate that the conclusion of this theorem may hold even though $\mathfrak{F C}$ is not normal. In $\$ 1$ a version of the Frobenius result is stated and the converse considered. This opens the question: What is the relationship between a group $\mathcal{G}$ and one of its subgroups $\mathfrak{H}$ if each irreducible $\mathcal{G}$-module over a field $\mathfrak{F}$ remains irreducible as an $\mathfrak{H}$-module? It is shown in $\$ 2$ that for "most" fields $\mathfrak{F}$ (the modular fields naturally cause a certain amount of difficulty) the answer is that $\mathcal{G}$ is an extension of $\mathfrak{H C}$ by an abelian group such that each conjugate class of $\mathcal{H C}$ is also a conjugate class of $\mathcal{G}$. To determine whether this last property leads to the conclusion that $\mathcal{G}$ is the trivial extension of $\mathfrak{H}$, extensions are considered in $\$ 3$ and it is shown that the answer is in general negative. However, using a result due to M. Hall [4] it is proved that this latter property does imply that $\mathcal{G}$ is the trivial extension of $\mathfrak{H C}$ in many cases.

Since results contingent on absolute irreducibility are used in certain proofs, ${ }^{1}$ it will be assumed throughout this note that $\mathfrak{F}$ is always a splitting field for every irreducible representation of the groups being discussed.

1. Preliminary remarks. Let $\mathfrak{H}$ be a subgroup of the finite group $\mathcal{G}$ and let $\mathfrak{M}$ be a left (right) $\mathcal{G}$-module with base field $\mathfrak{F}$. If $\mathfrak{N}$ is a left (right) $\mathfrak{H}$-submodule of $\mathfrak{M}$ and if $G \in \mathcal{G}$, then submodule $G \mathfrak{N}(\mathfrak{N} G)$ of $\mathfrak{M}$ is said to be a conjugate of $\mathfrak{N}$ relative to $\mathcal{G}$. Obviously it need not be an $\mathfrak{H}$-module.

Now the key to the Frobenius Theorem is the result [2]:

If $\mathfrak{H C}$ is a normal subgroup of $\mathcal{G}$ then an irreducible $\mathcal{G}$-module $\mathfrak{M}$ contains an irreducible $\mathfrak{H}$-submodule $\mathfrak{N}$ which has the property that each conjugate of $\mathfrak{R}$ relative to $\mathcal{G}$ is also an $\mathfrak{H}$-module.

Consideration of the converse proposition leads to the following:

Received by the editors February 20, 1958.

${ }^{1}$ It was pointed out by the referee that Theorem 3, for example, may be false if $\mathfrak{F}$ is not a splitting field for every irreducible representation of $G$ and $H$. The symmetric group on three elements, its normal subgroup, and the rational field illustrate this possibility. 
THEOREM 1. If Te is a subgroup of $\mathcal{G}$ such that each irreducible G-module $\mathfrak{M}$ over a field $\mathfrak{F}$ contains an irreducible $\mathfrak{H}-$-submodule $\mathfrak{U}$ all of whose conjugates relative to $\mathcal{G}$ are also $\mathfrak{H}$-modules, then each irreducible $\mathfrak{K}$-module remains irreducible as an $\mathfrak{H}$-module, where $\mathfrak{K}$ is the minimal normal subgroup of $\mathcal{G}$ which contains $\mathcal{H}$.

Let $\mathfrak{M}$ be an irreducible left $\mathcal{G}$-module. Since $\mathfrak{K}$ is normal in $\mathcal{G}, \mathfrak{M}$ is a direct sum of conjugate irreducible left $\mathfrak{K}$-modules, $\mathfrak{N}_{i}$, each of dimension $m$ relative to $\mathfrak{F}: \mathfrak{M}=\mathfrak{N}_{1}+\cdots+\mathfrak{N}_{n}, n \geqq 1$. On the other hand, from the hypothesis $\mathfrak{M}$ contains an irreducible left $\mathfrak{H}$-submodule $\mathfrak{U}$ all of whose conjugates relative to $\mathcal{G}$ are also $\mathfrak{H}$-modules, necessarily irreducible. Now let $G \in \mathcal{G}, H \in \mathcal{H}$; then $G \mathfrak{U}$ is an $\mathfrak{H}$-module and therefore $\left(G^{-1} H G\right) \mathfrak{U}=G^{-1} H(G \mathfrak{U})=G^{-1}(G \mathfrak{U})=\mathfrak{U}$. So $\mathfrak{U}$, of dimension $u$ over $\mathfrak{F}$, is an irreducible $\mathcal{K}$-module. Therefore $m=u$ and since each $\mathfrak{R}_{i}$ is also a left $\mathfrak{H}$-module it must remain irreducible as an $\mathfrak{H}$-module. As every irreducible $\mathfrak{K}$-module is $\mathcal{K}$-isomorphic with a submodule of a G-module, the result follows.

This interesting relationship between $\mathscr{K}$ and $\mathscr{K}$ will be investigated in the remainder of the paper.

2. Property g. To simplify matters we introduce the following definition. A subgroup $\mathcal{H C}$ of the group $G$ is said to possess property $g$ relative to the field $F$ if each irreducible $\mathcal{G}$-module over $\mathfrak{F}$ remains irreducible as an $\mathfrak{F}$-module.

THEOREM 2. If $\mathfrak{H C}$ possesses property $\mathrm{g}$ relative to $\mathfrak{F}$ then $\mathfrak{3 C}$ is normal in $\mathrm{G}$ and $\mathrm{G} / \mathcal{H C}$ is an abelian group if either of the following conditions is satisfied:

(i) The radical $\Re(\mathcal{S})$ of the group algebra $\mathfrak{A}(\mathcal{G})$ of $\mathcal{G}$ over $F$ equals $\mathfrak{A}(\mathcal{G}) \cdot \mathfrak{R}(\mathfrak{H C})$, where $\mathfrak{R}(\mathfrak{H C})$ is the radical of $\mathfrak{U}(\mathfrak{H C})$, the group algebra of $\mathfrak{H C}$ over $\mathfrak{F}$.

(ii) The characteristic of $\mathfrak{F}$ is $p$ and $\mathfrak{K C}$ is a Sylow p-subgroup of $\mathrm{G}$.

Let $\mathfrak{S}$ be the ideal of $\mathfrak{A}(\mathfrak{F C})$ which has as its basis the differences $H_{i}-H_{j}$, all $H_{i}, H_{j} \in \mathfrak{H C}$. Then $\mathfrak{H C}$ is normal in $g$ if and only if the left ideal $\mathfrak{l}=\mathfrak{A}(\mathfrak{G}) \cdot \mathfrak{H}$ is a two-sided ideal in $\mathfrak{A}(\mathcal{G})$. Now (i) implies that $\mathfrak{R} \Re(\mathcal{G})$ since $\mathfrak{S} \supseteq \Re(\mathfrak{K})$, so it will be sufficient to show that the image $\overline{\mathfrak{l}}$ of $\mathfrak{R}$ in $\overline{\mathfrak{A}}(\mathcal{G})=\mathfrak{A}(\mathcal{G})-\mathfrak{R}(\mathcal{G})$ is an ideal. $\overline{\mathfrak{A}}(\mathcal{G})$ contains an algebra $\mathfrak{D} \cong \overline{\mathfrak{Y}}(\mathfrak{K C})$ and $\mathfrak{D}=\mathfrak{U} \oplus \mathfrak{B}$ with $\mathfrak{U} \cong \mathfrak{H}(\mathfrak{F})-\mathfrak{S}$ and of dimension one over $\mathfrak{F}$. Then $\overline{\mathfrak{A}}(\mathcal{G})=\overline{\mathfrak{A}}(\mathcal{G}) \mathfrak{D}=\overline{\mathfrak{A}}(\mathcal{G}) \mathfrak{U}+\overline{\mathfrak{A}}(\mathcal{G}) \mathfrak{B}$, a direct sum of left ideals of $\overline{\mathfrak{A}}(\mathcal{G})$, with $\overline{\mathfrak{A}}(\mathcal{G}) \mathfrak{B} \cong \overline{\mathfrak{Q}}$. But $\overline{\mathfrak{A}}(\mathcal{G}) \mathfrak{U}$ and $\overline{\mathfrak{Z}}$ are right $\mathfrak{C}$-modules, so if $\mathfrak{B}$ is a minimal right ideal of $\mathfrak{A}(\mathcal{G})$, hence an irreducible right $\mathfrak{H}$-module, it must lie entirely in $\overline{\mathfrak{A}}(\mathcal{G}) \mathfrak{U}$ or $\overline{\mathfrak{R}}$. Hence $\overline{\mathfrak{R}}$ 
is also a right ideal of $\overline{\mathfrak{A}}(\mathcal{G})$ and so $\mathfrak{H C}$ is normal in $\mathcal{G}$. Furthermore $\mathcal{G} / \mathfrak{H C}$ is represented isomorphically over $\mathfrak{A}(\mathcal{G})-\mathfrak{Q} \cong \overline{\mathfrak{Y}}(\mathcal{G}) \mathfrak{U}$ which is necessarily a sum of fields since $\mathfrak{U}$ is one dimensional.

If (ii) is satisfied then all the irreducible representations of $G$ are one dimensional since the only irreducible representation of $\mathfrak{H C}$ is the identity representation. Therefore there exists a minimal normal subgroup $\mathscr{K}$ such that $\mathcal{G} / \mathcal{K}$ is abelian and $\mathfrak{A}(\mathcal{G} / \mathcal{K})$ is semisimple. It follows simply (cf. [3]) that $\mathcal{K}$ is necessarily of order $p^{a}$ and hence $\mathfrak{K}=\mathfrak{H}$.

If $\mathfrak{F}$ is restricted so that $\mathfrak{A}(\mathcal{G})$ is semisimple then the following deeper result may be obtained.

THEOREM 3. If $\mathfrak{H}$ is a subgroup of $\mathcal{G}$ possessing property $g$ relative to the field $\mathfrak{F}$ of characteristic 0 or $p,(p, o(G))=1$, then each conjugate class of $\mathcal{H}$ is also a conjugate class in $\mathrm{G}$.

Let $\mathfrak{S}(\mathcal{G})$ and $\mathfrak{S}(\mathfrak{H C})$ be the centers of $\mathfrak{A}(\mathcal{G})$ and $\mathfrak{A}(\mathfrak{H C})$ respectively. We must show that $\mathbb{S}(\mathfrak{H C})$ is a subalgebra of $\mathbb{E}(\mathcal{G})$. Let $\mathbb{R}$ be a minimal left ideal of $\mathfrak{A}(\mathcal{G})$; hence it is an irreducible left $\mathfrak{F}$-module and so there exists a primitive idempotent $e \in \mathfrak{S}(\mathfrak{H C})$ such that $e \mathfrak{A}(\mathfrak{H C}) \mathbb{R}=\mathbb{R}$. Now $\mathfrak{A}(\mathcal{G})=\mathfrak{A}(\mathfrak{F C}) \cdot \mathfrak{A}(\mathcal{G})$ and $e \mathfrak{H}(\mathfrak{H C}) \mathfrak{A}(\mathcal{G}) \supseteq \mathfrak{R}$, so if $\mathfrak{L}$ is the set of all minimal left ideals $\mathfrak{R}$ of $\mathfrak{A}(\mathcal{G})$ such that $e \mathfrak{A}(\mathfrak{H C}) \mathfrak{R}=\mathfrak{R}$, then $e \mathfrak{A}(\mathfrak{H C}) \mathfrak{A}(\mathcal{G})$

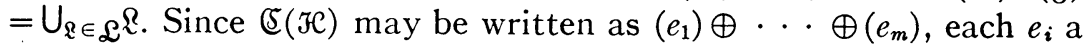
primitive idempotent, then $\mathfrak{A}(\mathcal{G})=e_{1} \mathfrak{A}(\mathfrak{H C}) \mathfrak{A}(\mathcal{G})+\cdots+e_{m} \mathfrak{A}(\mathfrak{H C}) \mathfrak{A}(\mathcal{G})$ is a direct decomposition of $\mathfrak{A}(\mathcal{G})$ into left ideals. Observing that $e_{i}-G e_{i} G^{-1}$ annihilates $\mathfrak{A}(\mathcal{G})$ from the left, any $G \in \mathcal{G}$, we conclude that $\mathbb{S}(\mathfrak{F}) \subset \mathbb{S}(\mathcal{G})$.

Indicative of the inconclusiveness of the modular case is

THEOREM 4. If $\mathfrak{H C}$ is a subgroup of $\mathrm{G}$ possessing property $\mathrm{g}$ over the field $\mathfrak{F}$ of characteristic $p$ and if all the irreducible representations of $\mathfrak{H C}$ over $\mathfrak{F}$ are one dimensional, then $\mathcal{G}$ is an extension of a p-group by an abelian group of order $q,(q, p)=1$. Conversely, if $\mathcal{G}$ is an extension of a proup by an abelian group of order $q,(q, p)=1$, then any subgroup $\mathfrak{H}$ of $\mathcal{G}$ possesses property 9 relative to a field of characteristic $p$.

Since an irreducible $\mathcal{H}$-module has dimension one, property $g \mathrm{im}$ plies that each irreducible $\mathcal{G}$-module is one dimensional over $\mathfrak{F}$. Therefore $\overline{\mathfrak{A}}(\mathcal{G})=\mathfrak{A}(\mathcal{G})-\mathfrak{R}(\mathcal{G})$ is a commutative algebra. If $\mathcal{G}^{\prime}$ is the commutator subgroup of $\mathcal{G}$ and if $\mathfrak{T}$ is the ideal of $\mathfrak{A}(\mathcal{G})$ generated by the differences $G_{i}-G_{j}$, all $G_{i}, G_{j} \in \mathcal{G}^{\prime}$, then clearly $\mathfrak{T} \subseteq \Re(\mathcal{S})$. This means that $\mathrm{g}^{\prime}$ is a p-group (cf. [3]), and the remainder of the theorem is obvious. 
Throughout the remainder of the paper the field $\mathfrak{F}$ will be assumed to have characteristic 0 or $p$ with $(p, g)=1, g$ the order of $g$. Then the next result completely characterizes property $\mathfrak{g}$ over $\mathfrak{F}$.

TheOREM 5. Let te be a normal subgroup of $\mathrm{g}$ of order $h$ and let $\mathrm{HC}$ contain $s \mathfrak{H}$-conjugate classes. Then $\mathfrak{H C}$ possesses property $\mathfrak{g}$ over $\mathfrak{F}$ if and only if $\mathrm{g}$ contains $n s \mathrm{G}$-conjugate classes, where $\mathrm{g}=\mathrm{hn}$.

Let $e$ be a primitive idempotent from the center of $\mathfrak{A}(\mathfrak{H C})$. Then $\mathfrak{T}=e \mathfrak{A}(\mathfrak{H C})$ is a minimal two-sided ideal of $\mathfrak{A}(\mathfrak{H C})$ of order $t^{2}$. If $\mathfrak{H C}$ possesses property $\mathscr{G}$, then by Theorem $3 e$ is a central idempotent of $\mathfrak{A}(\mathcal{G})$ and therefore $\mathfrak{B}=e \mathfrak{A}(\mathfrak{H}) \mathfrak{A}(\mathcal{G})$ is a two-sided ideal of $\mathfrak{A}(\mathcal{G})$ of order $n t^{2}$. Since $\mathfrak{T}$ is orthogonal with $\mathfrak{H}(\mathfrak{H C})-\mathfrak{T}$ it follows that each minimal $\mathfrak{H}$-submodule of $\mathfrak{B}$ is isomorphic with a minimal $\mathfrak{H}$-submodule of $\mathfrak{T}$ and hence is of order $t$. Then it follows from property $\mathfrak{g}$ that each minimal left or right ideal of $\mathfrak{B}$ is of order $t$, and therefore $\mathfrak{B}$ is expressible as a direct sum of $n$ two-sided ideals of $\mathfrak{U}(\mathcal{G})$, each of order $t^{2}$. Since the dimension of the center of $\mathfrak{A}(\mathfrak{H C})$ is $s$ this implies that $\mathfrak{A}(\mathcal{G})$ decomposes into a direct sum of $n s$ minimal ideals. Hence $\subseteq$ contains $n s$ conjugate classes.

Conversely, suppose $\mathcal{G}$ possesses $n s$ conjugate classes. Since $\mathcal{T}$ has $s$ conjugate classes, $\mathfrak{A}(\mathfrak{H C})=\mathfrak{I}_{1} \oplus \cdots \oplus \mathfrak{I}_{s}$ and this decomposition is unique. Now if $G \in \mathcal{G}, A \in \mathfrak{A}(\mathfrak{H C})$, the mapping $\theta_{G}: A \rightarrow A^{G}=G A G^{-1}$ is an automorphism of $\mathfrak{A}(\mathfrak{H C})$ and $\mathfrak{T}_{i}^{G}$ is a minimal ideal $\mathfrak{T}_{j}$ of $\mathfrak{A}(\mathfrak{H C})$. Therefore, under the set of all automorphisms induced by inner automorphisms of $\mathcal{G}$, the minimal ideals $\mathfrak{I}$ of $\mathfrak{A}(\mathfrak{H C})$ separate into nonoverlapping sets of transitivity, $S_{1}, \cdots, S_{m}$. That is, if $S_{i}$ consists of the ideals $\mathfrak{T}_{i, 1}, \cdots, \mathfrak{T}_{i, d(i)}$, then $\mathfrak{T}_{i j}^{G}=\mathfrak{T}_{i k}, 1 \leqq k \leqq d(i)$, for any $G \in \mathcal{G}$, and given any pair $\mathfrak{I}_{i p}$ and $\mathfrak{I}_{i q}$ there exists an element $G$ in $\mathcal{G}$ such that $\mathfrak{T}_{i q}=\mathfrak{T}_{i p}^{G}$. Then $\mathfrak{B}_{i}=\left(\mathfrak{I}_{i, 1}+\cdots+\mathfrak{I}_{i, d(i)}\right) \mathfrak{U}(\mathcal{G})$ is a two-sided ideal of $\mathfrak{A}(\mathcal{G})$ of order $n t_{i}^{2} d(i), t_{i}^{2}$ the order of $\mathfrak{T}_{i j}$.

Let $\mathfrak{R}$ be a minimal left ideal of $\mathfrak{B}_{i}$. Then $\mathfrak{T}_{i j} \mathfrak{R} \neq(0)$ for some $j$ and therefore, because of the transitivity of $S_{i}$, for all $j$. Since $\mathfrak{I}_{\imath j} \mathfrak{R}$ is necessarily of order $\geqq t_{i}$ and since $\mathfrak{I}_{i j} \mathfrak{I}_{i p}=\delta_{j q} \mathfrak{I}_{i j}$, this implies that the order of $\mathfrak{Q}$ is $\geqq t_{i} d(i)$. Therefore a minimal two-sided ideal of $\mathfrak{B}_{\boldsymbol{i}}$ is of order $\geqq t_{i}^{2}[d(i)]^{2}$, and so no decomposition of $\mathfrak{B}_{i}$ contains more than $n / d(i)$ two-sided ideals. Therefore $\mathfrak{A}(\mathcal{G})$ decomposes into a sum of not more than $n(1 / d(1)+\cdots+1 / d(m))$ minimal ideals. However, since $\mathcal{G}$ contains $n s$ conjugate classes, $\mathfrak{A}(\mathcal{G})$ decomposes into a direct sum of $n s$ minimal ideals. Hence $d(1)=\cdots=d(m)=1, m=s$, and each minimal left ideal $\mathfrak{R}$ of $\mathfrak{B}_{i}$ is of order $t_{i}$. Since $\mathfrak{R}$ is a left $\mathfrak{I}_{i}$-module whose order equals the order of a minimal left ideal of $\mathfrak{T}_{i}$ it follows that $\mathfrak{H C}$ possesses property $\mathfrak{g}$. 
Berman has proved [1] that if $\mathcal{H C}$ is a normal subgroup of $\mathcal{G}$ such that $\mathrm{G} / \mathcal{H C}$ is cyclic of order $n$ and if each of $s \mathcal{G}$-conjugate classes $C_{i}$ contained in $\mathfrak{H C}$ splits into $h_{i} \mathfrak{H C}$-conjugate classes, then $\mathcal{G}$ contains $n\left(h_{1}^{-1}+\cdots+h_{s}^{-1}\right)$ conjugate classes. This result and the previous theorem yield a partial converse to Theorem 3:

THEOREM 6. If $\mathcal{G}$ is an extension of $\mathfrak{H C}$ by a cyclic group and if each conjugate class of $\mathfrak{H C}$ is also a conjugate class of $\mathrm{G}$, then $\mathfrak{H C}$ possesses property $\mathfrak{g}$ over $\mathfrak{F}$.

3. Group extensions by abelian groups. Obviously the trivial extension $\mathcal{G}$ of a group $\mathcal{H}$ by an abelian group $Q, \mathcal{G}=\mathcal{H} \times \mathcal{Q}$, contains a normal subgroup $\mathcal{H}^{\prime} \cong \mathcal{H}$ possessing property $\mathscr{g}$ over $F$. Is the trivial extension the only one for which this is so? We shall see that the answer to this depends on whether or not the order $c$ of $\mathfrak{F}$ is prime to the order $n$ of $\mathrm{G} / \mathfrak{F C}$.

If $\mathfrak{H}$ possesses property $g$ in $\mathcal{G}$ then we have seen that $\mathfrak{H}$ is normal

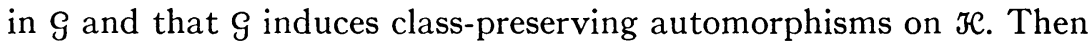
the additional condition, $(c, n)=1$, permits us to apply a result due to M. Hall [4, Theorem 6.1] and to conclude that $g$ is a trivial extension of $\mathfrak{F C}$.

In the other direction we prove the following:

LEMMA. If $\mathfrak{H}$ is a group containing a q-subgroup $a, q$ a prime, in its center, then there exists a nontrivial extension $\mathrm{G}$ of $\mathfrak{H}$ such that $\mathrm{G}$ contains a subgroup $\mathfrak{H}^{\prime} \cong \mathscr{H}$ possessing property $\mathcal{G}$ in $\mathcal{G}, \mathcal{G} / \mathfrak{H C}^{\prime}$ of order $q$.

Let $A$ be a generator of a cyclic $q$-subgroup of FC which is of maximal order $q^{r}$ among those contained in the center of $\mathfrak{H C}$. Let $x$ be an indeterminate and define $\mathcal{G}$ to be the set of all ordered pairs $\left(x^{i}, H\right)$ where $0 \leqq i<q, x^{0}=1$, and $H$ is an element of $\mathfrak{F}$. Then multiplication in $\mathrm{G}$ is determined by the following definitions: $\left(x, H_{0}\right)^{q}$ $=(1, A)$, where $H_{0}$ is the identity element of $\mathfrak{F}$, and $\left(x^{i}, H_{j}\right)\left(x^{k}, H_{n}\right)$ $=\left(x^{m}, A^{t} H_{j} H_{n}\right)$ where $i+j=m+t q, 0 \leqq m<q$. It is easy to verify that $\mathcal{G}$ is a group containing a subgroup $\mathfrak{F}^{\prime}=(1, \mathfrak{H}) \cong \mathscr{H}$ possessing property $\mathscr{g}$ in $\mathcal{G}$. Furthermore $\mathcal{G}$ is not isomorphic with the trivial extension of $\mathcal{H}$ since it contains a cyclic $q$-subgroup of order $q^{r+1}$ in its center.

To summarize these results:

THEOREM 7. If a subgroup $\mathfrak{F}$ of a group $\mathcal{G}$ possesses property $\mathfrak{g}$ relative to $\mathfrak{F}$ then $\&$ may be a nontrivial extension of $\mathfrak{F}$ but only if the order of $\mathcal{G} / \mathcal{H}$ is not prime to the order of $\mathcal{H}$. 


\title{
REFERENCES
}

1. S. D. Berman, Group algebras of Abelian extensions of finite groups, Dokl. Akad. Nauk. SSSR (N.S.) vol. 102 (1955) pp. 431-434; Math. Rev. vol. 17 (1956) p. 235.

2. A. H. Clifford, Representations induced in invariant subgroups, Ann. of Math. vol. 38 (1937) pp. 533-548.

3. W. E. Deskins, Finite Abelian groups with isomorphic group algebras, Duke Math. J. vol. 23 (1956) pp. 35-40.

4. M. Hall, Group rings and extensions, Ann. of Math. vol. 38 (1938) pp. 220-234.

Michigan State University

\section{A RING ADMITTING MODULES OF LIMITED DIMENSION}

\author{
WILLIAM G. LEAVITT
}

Let $K$ be a ring with unit. A module ${ }^{1} M$ over $K$ is said to be finite dimensional if it (i) is finitely based, and (ii) contains no infinite independent set. For such a module there must exist [1, Theorem 7 , p. 245] an integer $n$ such that all bases have length $n$ (the invariant basis number property), and no independent set has length greater than $n$. It was shown in a recent paper $[1$, Theorem 6, p. 245] that this property carries downward with decreasing length of basis. That is: If $K$ admits a module of finite dimension $n$, then every module over $K$ having a basis of length $\leqq n$ is also finite dimensional.

It was remarked (in [1]) that this leaves open the possibility that a ring could exist admitting only modules of limited dimension. That is, for some fixed integer $n$ there might exist a ring $K$ such that a module over $K$ is finite dimensional if and only if it has a basis of length $\leqq n$. It is the purpose of this paper to construct such a ring for arbitrary $n$.

Let $R$ be the ring of (noncommutative) polynomials generated over the field of integers modulo 2 by a countably infinite set of symbols $\left\{x_{i}, y_{j}\right\}$, with $i=1, \cdots, m=(n+2)(n+1) ; j=1,2, \cdots$, where $n$ is the fixed integer chosen. Let $R^{\prime}$ be the subring of $R$ generated by the $\left\{x_{i}\right\}$. It is desired to order a (suitably restricted) set of $n$-dimensional row vectors of members of $R^{\prime}$. Begin by ordering the set of all

Received by the editors March 11, 1957 and, in revised form, February 28, 1958.

1 Throughout this paper "module" will mean "left module." 